\title{
HYBRID MATRIX MODELS AND THEIR POPULATION DYNAMIC CONSEQUENCES*
}

\author{
SANYI TANG ${ }^{1}$
}

\begin{abstract}
In this paper, the main purpose is to reveal what kind of qualitative dynamical changes a continuous age-structured model may undergo as continuous reproduction is replaced with an annual birth pulse. Using the discrete dynamical system determined by the stroboscopic map we obtain an exact periodic solution of system with density-dependent fertility and obtain the threshold conditions for its stability. We also present formal proofs of the supercritical flip bifurcation at the bifurcation as well as extensive analysis of dynamics in unstable parameter regions. Above this threshold, there is a characteristic sequence of bifurcations, leading to chaotic dynamics, which implies that the dynamical behavior of the single species model with birth pulses are very complex, including small-amplitude annual oscillations, large-amplitude multi-annual cycles, and chaos. This suggests that birth pulse, in effect, provides a natural period or cyclicity that allows for a period-doubling route to chaos. Finally, we discuss the effects of generation delay on stability of positive equilibrium (or positive periodic solution), and show that generation delay is found to act both as a destabilizing and a stabilizing effect.
\end{abstract}

Mathematics Subject Classification. 58J90, 92D40.

Received: June 24, 2003.

\section{INTRODUCTION}

For many animal species (crustacean, insects, amphibians, ...), the individuals take on different morphological shapes before reaching their final adult state. This multiplicity of developmental stages gives rise to individuals with a complex life cycle. Copepods are small crustacean whose molting processes determine the succession of the different stages. These different stages are characterized by different shapes, sizes and behaviors, that is, the vital rates(rates of survival, development, and reproduction) almost always depend on age, size, or development stage, but above all they play very different roles in marine ecosystems.

Stage structure models have received much attention in recent years $[2,3,6,7,14,16,17,28,29,36,37]$. This is not only because they are much more simple than the models governed by partial differential equations but also they can exhibit phenomena similar to those of partial differential models [5], and many important physiological parameters can be incorporated.

Population models fall into two fundamental categories: discrete and continuous. In the discrete category, Leslie matrix models constitute the basic class of age-structured models. Mckendrick partial differential equation

\footnotetext{
Keywords and phrases. Hybrid matrix model, birth pulse, supercritical flip bifurcation, stroboscopic map, generation delay.

* This work is supported by National Natural Science Foundation of China.

1 Institute of Mathematics, Academy of Mathematics and System Sciences, Academia Sinica, Beijing, 100080, P.R. China.

e-mail: tsy@math08.math.ac.cn
} 
models and nonlinear differential systems studied in $[6,7]$ play the same role in the category of continuous models. Many authors have studied the mathematical connections between these two famous classes of models $[19,36,37]$.

These models have invariably assumed that the mature population reproduce throughout the year, whereas it is often the case that births are seasonal or occur in regular pulses. The continuous reproduction of mature population is then removed from the model, and replaced with an annual birth pulse. These models are subject to short-term perturbations which are often assumed to be in the form of impulses in the modeling process. Consequently, impulsive differential equations (hybrid dynamical systems) provide a natural description of such systems $[4,26]$. Equations of this kind are found in almost every domain of applied sciences. Numerous examples are given in Bainov's and his collaborators' books [4]. They generally describe phenomena which are subject to steep and/or instantaneous changes. Some impulsive equations have been recently introduced in population dynamics in relation to: vaccination $[1,35]$, and chemotherapeutic treatment of disease $[25,34]$. The main question which we consider here is to reveal what kind of qualitative dynamical changes a continuous agestructured model will undergo as the continuous reproduction is replaced with an annual birth pulse. More specific, we will answer the following interesting questions:

(1) What are main difference on dynamical behavior between the hybrid matrix models and the continuous age-structured models described by a set of ordinary differential equations?

(2) What are the global dynamics of the hybrid matrix models depending on parameters?

(3) How do the generation delay and density-dependent fertility affect the dynamical behavior of hybrid matrix models?

In order to realize these goals, we shall investigate the dynamical properties of hybrid matrix models derived from the continuous age-structured models with birth pulse (i.e., the continuous reproduction of population is replaced with an annual birth pulse). Further, we restrict our attention to the two-dimensional hybrid matrix models(that is, the population is divided into immature and mature classes). In Section 4, we use the stroboscopic map, where the map determines the number of immature population and mature population, immediately after each pulse birth at the discrete times $m$ ( $m$ is a positive integer). When the birth rates of population are influenced by the population density, the discrete dynamical system determined by the stroboscopic map becomes nonlinear. The population in the pulsed birth time is characterized not by an exponential growth rate, but by the existence and stability of equilibria, by the supercritical flip bifurcations that occur when stability is lost, and by the patterns of dynamics (cycles, chaos) that follow the bifurcations. Further we focus our attention on the relationships between the differential dynamical system with birth pulses and the discrete dynamical system determined by the corresponding stroboscopic map. It is shown that dynamical behaviors of models with birth pulses are very complex, and include small-amplitude annual oscillations, large amplitude multi annual cycles, and chaos. That is, birth pulse, in effect, provides a natural period or cyclicity that allows for a period-doubling route to chaos. In the end we discuss how the regions in which the periodic solution of impulsive system is existent and stable change as parameters vary by using theoretical and numerical methods, and then obtain that the generation time delay has both stabilizing and unstabilizing effects. The population fluctuations associated with stage structure effects have been found by many authors [14,32]. For Nicholson's Blowflies, the period of the cycles is 2-3 times the maturation time, and for the Lawton's Plodia, the irregular fluctuations have a dominant period close to the generation time.

\section{Derivation of the MODEL}

Stage-structured models enter in the framework of these models for which qualitative behavior can be entirely determined by the sign of the Jacobian matrix: the dynamics of stage $i$ is the balance an input term in the stage depending on the stage $i-1$ (recruitment) and an output term (mortality, transfer to the next stage, dilution) depending on the stage $i$. Hence, the dynamics of each variable only depend on the variable itself and on the precedent one so that the system has a so-called loop structure $[6,17]$. Some stages may have more complex dynamics. For example in the classical models of age-structured populations of fishes, several stages of fish can 
spawn [24,28]. Such models, which are continuous Leslie models do not have a loop structure: the dynamics of the eggs depend on all the mature stages.

In general, we assume that a population with overlapping generations is subdivided into $n$ stages. All stages can be fertile and contribute to the newborn class through the birth rates $\beta_{j}$ (if there are $k$ juvenile classes, then $\beta_{1}=\beta_{2}=\cdots=\beta_{k}=0$ ). As time elapses, individuals in stage $i$ die with $\mu_{i}$ and survivors move to the next stage with transition rate or maturity rate $m_{i}$. This leads to the set of ordinary differential equations

$$
\left\{\begin{aligned}
\frac{\mathrm{d} A_{1}(t)}{\mathrm{d} t} & =\sum_{j=1}^{n} \beta_{j} A_{j}(t)-\left(m_{1}+\mu_{1}\right) A_{1}(t) \\
\frac{\mathrm{d} A_{2}(t)}{\mathrm{d} t} & =m_{1} A_{1}-\left(m_{2}+\mu_{2}\right) A_{2}(t) \\
& \vdots \\
\frac{\mathrm{d} A_{n-1}(t)}{\mathrm{d} t} & =m_{n-2} A_{n-2}-\left(m_{n-1}+\mu_{n-1}\right) A_{n-1}(t) \\
\frac{\mathrm{d} A_{n}(t)}{\mathrm{d} t} & =m_{n-1} A_{n-1}-\mu_{n} A_{n}(t)
\end{aligned}\right.
$$

Where $A_{i}(i=1,2, \cdots, n)$ represent biomass or number in stage $i$. It is assumed that $A_{j}(0) \geq 0, \sum_{j=0}^{n} A_{j}(0)>0$, and $\mu_{j}>0, j=1,2, \cdots, n$. The birth rates $\beta_{j}$, the transition rates $m_{j}$, and the death rates $\mu_{j}$ can be time dependent and density dependent in which case they become functions of one or more the class densities $A_{i}$. Populations with density-dependent fertility or survival rates have been extensively analyzed [27-30].

If the continuous reproduction of mature population is replaced with an annual birth pulse, then we have the following impulsive differential equations (2.2) and (2.3)

$$
\begin{aligned}
& \left\{\begin{array}{rl}
\frac{\mathrm{d} A_{1}(t)}{\mathrm{d} t} & =-\left(m_{1}+\mu_{1}\right) A_{1}(t), \\
\frac{\mathrm{d} A_{2}(t)}{\mathrm{d} t} & =m_{1} A_{1}-\left(m_{2}+\mu_{2}\right) A_{2}(t), \\
& \vdots \\
\frac{\mathrm{d} A_{n-1}(t)}{\mathrm{d} t} & =m_{n-2} A_{n-2}-\left(m_{n-1}+\mu_{n-1}\right) A_{n-1}(t), \\
\frac{\mathrm{d} A_{n}(t)}{\mathrm{d} t} & =m_{n-1} A_{n-1}-\mu_{n} A_{n}(t) .
\end{array} \quad t \neq k \in N,\right. \\
& \left\{\begin{array}{l}
A_{1}\left(k^{+}\right)= \\
A_{1}(k)+\sum_{j=1}^{n} \beta_{j} A_{j}(k),
\end{array}\right. \\
& A_{i}\left(k^{+}\right)=A_{i}(k), \quad i=2,3, \cdots, n,
\end{aligned}
$$

If we denote $A=\left(A_{1}, A_{2}, \cdots, A_{n}\right)^{T}$,

$$
M=\left(\begin{array}{cccccc}
-m_{1}-\mu_{1} & 0 & 0 & \cdots & 0 & 0 \\
m_{1} & -m_{2}-\mu_{2} & 0 & \cdots & 0 & 0 \\
\ldots & \cdots & \cdots & \cdots & \cdots & \cdots \\
0 & 0 & 0 & \cdots & -m_{n-1}-\mu_{n-1} & 0 \\
0 & 0 & 0 & \cdots & m_{n-1} & -\mu_{n}
\end{array}\right)
$$


and

$$
I=\left(\begin{array}{cccccc}
1+\beta_{1} & \beta_{2} & \beta_{3} & \cdots & \beta_{n-1} & \beta_{n} \\
0 & 1 & 0 & \cdots & 0 & 0 \\
\cdots & \cdots & \cdots & \cdots & \cdots & \cdots \\
0 & 0 & 0 & \cdots & 1 & 0 \\
0 & 0 & 0 & \cdots & 0 & 1
\end{array}\right)
$$

then systems (2.2) and (2.3) become the following hybrid matrix models

$$
\left\{\begin{array}{l}
\frac{\mathrm{d} A(t)}{\mathrm{d} t}=M A(t), \quad t \neq k \in N, \\
A\left(k^{+}\right)=I A(k), \quad t=k \in N .
\end{array}\right.
$$

\section{Positivity And Stroboscopic Map of System (2.4)}

From a biological point of view, population size should not be negative, that is, $A_{j}$ must be always nonnegative. For the hybrid matrix model (2.4), we have the following result on the positivity of solutions.

Lemma 3.1. The set $\Omega=\left\{0<A_{j}<\infty, j=1,2, \cdots, n\right\}$ is positive invariant for system (2.4). That is, the trajectories of (2.4) are always positive for all $0<t<\infty$ provided $A_{j}(0)>0$ for all $j$.

Proof. Since

$$
A_{j}(t)=\exp \left(-\int_{0}^{t}\left(m_{j}+\mu_{j}\right) \mathrm{d} r\right)\left[A_{j}(0)+\int_{0}^{t} m_{j-1} A_{j-1}(s) \exp \left(\int_{0}^{s}\left(m_{j}+\mu_{j}\right) \mathrm{d} u\right) \mathrm{d} s\right], \quad j=2,3, \cdots, n
$$

where $m_{n}=0$. If there is a time $t_{(j)}^{*}>0$ such that $A_{j}\left(t_{(j)}^{*}\right)=0$ and $A_{j}(t)>0$ for all $t<t_{(j)}^{*}$ and some $j$, but $A_{i}(t)>0$ for all $t \leq t_{(j)}^{*}$ and all $i>j$, then there exists a time interval $0<T_{j-1}<\bar{T}_{j-1} \leq t_{(j)}^{*}$ such that $A_{j-1}(t)<0$ for all $t \in\left(T_{j-1}, \bar{T}_{j-1}\right)$. Hence, there is a time $t_{(j-1)}^{*}<t_{(j)}^{*}$ such that $A_{j-1}\left(t_{(j-1)}^{*}\right)=0$. Continuing in this manner, there is a time $t_{1}^{*}>0$ such that $A_{1}\left(t_{1}^{*}\right)=0$ and $A_{i}(t)>0$ for all $t \leq t_{1}^{*}, i \geq 2$. However, from system (2.2) and (2.3) we have

$$
\begin{cases}\frac{\mathrm{d} A_{1}(t)}{\mathrm{d} t}=-\left(m_{1}+\mu_{1}\right) A_{1}(t), & t \neq k \in N, 0 \leq t \leq t_{1}^{*}, \\ A_{1}\left(k^{+}\right) \geq A_{1}(k)+\beta_{1} A_{1}(k), & t=k \in N, 0 \leq t \leq t_{1}^{*} .\end{cases}
$$

By the impulsive differential inequality [4], we have

$$
A_{1}\left(t_{1}^{*}\right) \geq A_{1}(0) \prod_{1}^{k_{1}}\left(1+\beta_{1}\right) \exp \left(\int_{0}^{t_{1}^{*}}-\left(m_{1}+\mu_{1}\right) \mathrm{d} r\right)>0,
$$

where $k_{1} \in N$ such that $t_{1}^{*} \in\left(k_{1}, k_{1}+1\right)$. This contradicts with $A_{1}\left(t_{1}^{*}\right)=0$ and completes the proof.

Remark. For stage-structured models, there may be no individuals in a life cycle stage or age class and the population stilll not be extinct. In fact, if $A_{j}(0) \geq 0$ and $\sum_{j=0}^{n} A_{j}(0)>0$, then we have $\sum_{j=0}^{n} A_{j}(t)>0$ for all $t \geq 0$.

In the rest of this paper, we assume that $m_{i}, \mu_{i}$ are constants. To this end of this section, we will obtain the analytical solutions of system (2.2) between the pulses which will be used to deduce the stroboscopic map of 
hybrid matrix models (2.4). System (2.2) can be written as the general form of a linear equation between the pulses

where $m_{0}=m_{n}=0$.

$$
\frac{\mathrm{d} A_{i}(t)}{\mathrm{d} t}+\left(m_{i}+\mu_{i}\right) A_{i}(t)=m_{i-1} A_{i-1}(t), \quad i=1,2, \cdots, n, \quad t \in[k, k+1),
$$

Equation (3.2) is solved by multiplying both sides by an integrating factor

$$
\phi(t)=\mathrm{e}^{\left(m_{i}+\mu_{i}\right)(t-k)}, \quad k \leq t<k+1 .
$$

The solution is

$$
A_{i}(t)=m_{i-1} \mathrm{e}^{-\left(m_{i}+\mu_{i}\right)(t-k)} \int_{k}^{t} A_{i-1}(s) \mathrm{e}^{\left(m_{i}+\mu_{i}\right)(s-k)} \mathrm{d} s+A_{i}(k) \mathrm{e}^{-\left(m_{i}+\mu_{i}\right)(t-k)} .
$$

We use integration by parts to evaluate the integral

$$
\int_{k}^{t} A_{i-1}(s) \mathrm{e}^{\left(m_{i}+\mu_{i}\right)(s-k)} \mathrm{d} s=\left[\frac{\mathrm{e}^{\left(m_{i}+\mu_{i}\right)(t-k)}}{m_{i}+\mu_{i}} A_{i-1}(t)-\frac{A_{i-1}(k)}{m_{i}+\mu_{i}}\right]-\int_{k}^{t} \frac{\mathrm{e}^{\left(m_{i}+\mu_{i}\right)(s-k)}}{m_{i}+\mu_{i}} \frac{\mathrm{d} A_{i-1}(s)}{\mathrm{d} s} \mathrm{~d} s,
$$

using (3.2) to substitute the derivative on the right-hand side

$$
\begin{aligned}
\int_{k}^{t} A_{i-1}(s) \mathrm{e}^{\left(m_{i}+\mu_{i}\right)(s-k)} \mathrm{d} s=\left[\frac{\mathrm{e}^{\left(m_{i}+\mu_{i}\right)(t-k)}}{m_{i}+\mu_{i}} A_{i-1}(t)\right. & \left.-\frac{A_{i-1}(k)}{m_{i}+\mu_{i}}\right] \\
& -\int_{k}^{t} \frac{\mathrm{e}^{\left(m_{i}+\mu_{i}\right)(s-k)}}{m_{i}+\mu_{i}}\left[m_{i-2} A_{i-2}(s)-\left(m_{i-1}+\mu_{i-1}\right) A_{i-1}(s)\right] \mathrm{d} s
\end{aligned}
$$

leading to a recurrence relation for the integral

$$
\begin{aligned}
\int_{k}^{t} A_{i-1}(s) \mathrm{e}^{\left(m_{i}+\mu_{i}\right)(s-k)} \mathrm{d} s & \\
\frac{1}{\left(m_{i}+\mu_{i}\right)-\left(m_{i-1}+\mu_{i-1}\right)} & {\left[\mathrm{e}^{\left(m_{i}+\mu_{i}\right)(t-k)} A_{i-1}(t)-A_{i-1}(k)-m_{i-2} \int_{k}^{t} A_{i-2}(s) \mathrm{e}^{\left(m_{i}+\mu_{i}\right)(s-k)} \mathrm{d} s\right] . }
\end{aligned}
$$

Substituting into $(3.3)$

$$
\begin{aligned}
A_{i}(t)= & \frac{m_{i-1}}{\left(m_{i}+\mu_{i}\right)-\left(m_{i-1}+\mu_{i-1}\right)}\left[A_{i-1}(t)-A_{i-1}(k) \mathrm{e}^{-\left(m_{i}+\mu_{i}\right)(t-k)}\right] \\
& -\frac{m_{i-1}}{\left(m_{i}+\mu_{i}\right)-\left(m_{i-1}+\mu_{i-1}\right)} \frac{m_{i-2}}{\left(m_{i}+\mu_{i}\right)-\left(m_{i-2}+\mu_{i-2}\right)}\left[A_{i-2}(t)-A_{i-2}(k) \mathrm{e}^{-\left(m_{i}+\mu_{i}\right)(t-k)}\right] \\
& \left.+\cdots+(-1)^{i} \prod_{j=1}^{i-1} \frac{m_{i-j}}{\left(m_{i}+\mu_{i}\right)-\left(m_{i-j}+\mu_{i-j}\right)} A_{1}(t)-A_{1}(k) \mathrm{e}^{-\left(m_{i}+\mu_{i}\right)(t-k)}\right] \\
& +A_{i}(k) \mathrm{e}^{-\left(m_{i}+\mu_{i}\right)(t-k)}
\end{aligned}
$$

or, in a more compact form

$$
\begin{aligned}
A_{i}(t)=\sum_{l=1}^{i-1}\left\{\prod_{j=1}^{l}(-1)^{l+1} \frac{m_{i-j}}{\left(m_{i}+\mu_{i}\right)-\left(m_{i-j}+\mu_{i-j}\right)}\left[A_{i-l}(t)-\mathrm{e}^{-\left(m_{i}+\mu_{i}\right)(t-k)} A_{i-l}(k)\right]\right\} & \\
& +A_{i}(k) \mathrm{e}^{-\left(m_{i}+\mu_{i}\right)(t-k)}, \quad i=1,2, \cdots, n .
\end{aligned}
$$


The equation of (3.4) shows that the proportion of individuals at time $t \in[k, k+1)$ in class $i$ is thus given by the term $A_{i}(k) \mathrm{e}^{-\left(m_{i}+\mu_{i}\right)(t-k)}$, representing the decay of the proportion since the time of birth pulse before $t$, $A_{i}(k)$, plus a dynamical term representing the contribution of class $i-l$ to class $i$ (the factor in square brackets), weighted by a quotient of outflow rates.

Since equation (3.4) holds between pulses. At each successive pulse, more class $A_{1}$ is added. If we denote

$$
\begin{array}{r}
\bar{A}_{i}(k) \doteq \sum_{l=1}^{i-1}\left\{\prod_{j=1}^{l}(-1)^{l+1} \frac{m_{i-j}}{\left(m_{i}+\mu_{i}\right)-\left(m_{i-j}+\mu_{i-j}\right)}\left[\bar{A}_{i-l}(k)-\mathrm{e}^{-\left(m_{i}+\mu_{i}\right)} A_{i-l}(k)\right]\right\} \\
+A_{i}(k) \mathrm{e}^{-\left(m_{i}+\mu_{i}\right)}, \quad i=1,2, \cdots, n,
\end{array}
$$

then we can obtain the following stroboscopic map for hybrid matrix model (2.4), i.e.,

$$
\left\{\begin{array}{l}
A_{1}(k+1)=A_{1}(k) \mathrm{e}^{-\left(\mu_{1}+m_{1}\right)}+\sum_{i=1}^{n} \beta_{i} \bar{A}_{i}(k), \\
A_{j}(k+1)=\bar{A}_{j}(k), \quad j=2,3, \cdots, n
\end{array}\right.
$$

Example. If $n=2,(3.4)$ gives

$$
\left\{\begin{array}{l}
A_{1}(t)=A_{1}(k) \mathrm{e}^{-\left(m_{1}+\mu_{1}\right)(t-k)} \\
A_{2}(t)=\frac{m_{1}}{\mu_{2}-\left(m_{1}+\mu_{1}\right)}\left[A_{1}(t)-A_{1}(k) \mathrm{e}^{-\mu_{2}(t-k)}\right]+A_{2}(k) \mathrm{e}^{-\mu_{2}(t-k)}, k \leq t<k+1
\end{array}\right.
$$

Then we have

$$
\left\{\begin{array}{l}
\bar{A}_{1}(k)=A_{1}(k) \mathrm{e}^{-\left(m_{1}+\mu_{1}\right)} \\
\bar{A}_{2}(k)=\frac{m_{1}}{\mu_{2}-\left(m_{1}+\mu_{1}\right)}\left[\bar{A}_{1}(k)-A_{1}(k) \mathrm{e}^{-\mu_{2}}\right]+A_{2}(k) \mathrm{e}^{-\mu_{2}}
\end{array}\right.
$$

Therefore, by (3.5), we obtain the following stroboscopic map for two-dimensional hybrid matrix model.

$$
\left\{\begin{array}{l}
A_{1}(k+1)=A_{1}(k) \mathrm{e}^{-\left(\mu_{1}+m_{1}\right)}+\sum_{i=1}^{2} \beta_{i} \bar{A}_{i}(k), \\
A_{2}(k+1)=\bar{A}_{2}(k)
\end{array}\right.
$$

\section{Dynamical Behavior for two generation CASE}

The results of our two-dimensional analysis are presented in two separate parts. In the first parts, 4.1, we study the effects of density-dependent fertility without generation delay on the stroboscopic map which determined the state after a pulse in terms of the state after the previous pulse. Further, we discuss the relationships between the impulsive differential dynamical system and the discrete dynamical system determined by the corresponding stroboscopic map. In the second, 4.2 , the combined effect of density-dependent fertility and generation delay are investigated. In each case, we assume that $\beta_{i}(i=1,2)$ are density-dependent in which the density dependence is through a dependence on a weighted total population size, that is, $\beta_{i}=b_{i} \mathrm{e}^{-\left(A_{1}(t)+A_{2}(t)\right)}$ where $b_{i}(i=1,2)$ are constants. 


\subsection{The case of no reproductive delay}

In this section we study the two-dimensional hybrid matrix model

$$
\left\{\begin{array}{l}
\frac{\mathrm{d} A_{1}(t)}{\mathrm{d} t}=-\left(m_{1}+\mu_{1}\right) A_{1}(t) \\
\frac{\mathrm{d} A_{2}(t)}{\mathrm{d} t}=m_{1} A_{1}(t)-\mu_{2} A_{2}(t), \quad t \neq k \in N \\
A_{1}\left(k^{+}\right)=A_{1}(k)+\left[b_{1} A_{1}(k)+b_{2} A_{2}(k)\right] \mathrm{e}^{-\left(A_{1}(k)+A_{2}(k)\right)} \\
A_{2}\left(k^{+}\right)=A_{2}(k), \quad t=k \in N
\end{array}\right.
$$

For the simplicity, we assume $\mu_{1}=\mu_{2}=\mu$ and $b_{1}=b_{2}=b$. From $(3.7)$, we have $\bar{A}_{1}(k)+\bar{A}_{2}(k)=\mathrm{e}^{-\mu}\left(A_{1}(k)+\right.$ $\left.A_{2}(k)\right)$, and by (3.8), the following stroboscopic map of (4.1) is obtained.

$$
\left\{\begin{array}{l}
A_{1}(k+1)=b p\left[A_{1}(k)+A_{2}(k)\right] \mathrm{e}^{-p\left(A_{1}(k)+A_{2}(k)\right)}+p q A_{1}(k), \\
A_{2}(k+1)=p(1-q) A_{1}(k)+p A_{2}(k) .
\end{array}\right.
$$

where $p=\mathrm{e}^{-\mu}, q=\mathrm{e}^{-m_{1}}$.

Let $x=p A_{1}, y=p A_{2}$, then system (4.2) becomes as follows

$$
\left\{\begin{array}{l}
x_{k+1}=b p\left[x_{k}+y_{k}\right] \mathrm{e}^{-\left(x_{k}+y_{k}\right)}+p q x_{k} \\
y_{k+1}=p(1-q) x_{k}+p y_{k}
\end{array}\right.
$$

The dynamics of the nonlinear model (4.3) can be studied as a function of any of the parameters. We will focus here on $b$ and document the changes in the qualitative dynamics of the model (4.3) as $b$ varies. First, the trivial equilibrium $E_{0}(0,0)$ is always a solution to equation (4.3). When $b$ is small enough, this solution is locally stable, and the species cannot increase when rare or invade a habitat from which it is absent. Our first concern will be with the conditions under which $E_{0}(0,0)$ becomes unstable, permitting colonization of the population. Second, the destabilization of $E_{0}$ with increasing $b$ is always accompanied by the appearance of a stable positive equilibrium $E^{*}$. As $b$ is increased further, this equilibrium in turn becomes unstable. A supercritical flip bifurcation occurs and the equilibrium loses stability to a stable two-cycle. Finally, as $b$ is increased still further, there is a characteristic sequence of bifurcations, leading, in most cases, to chaotic dynamics.

\subsection{Bifurcations of $(\boldsymbol{x}, \boldsymbol{y})=(\mathbf{0}, \mathbf{0})$}

In the neighborhood of $(x, y)=(0,0)$, the dynamics of equation $(4.3)$ is controlled by the linearization

$$
X_{m+1}=B X_{m}
$$

with $B$ as in the linear counterpart of (4.3) and $X=(x, y) . X=0$ is stable when the eigenvalues of $B$ are less than one in magnitude. This is true only when $B$ satisfies the three Jury conditions [21]:

$$
\begin{gathered}
1-\operatorname{tr} B+\operatorname{det} B>0, \\
1+\operatorname{tr} B+\operatorname{det} B>0, \\
1-\operatorname{det} B>0 .
\end{gathered}
$$

These three conditions correspond to the three ways that an eigenvalue may exit the unit circle in the complex plane. If inequality (4.5a) is violated, then one of the eigenvalues of $B$ is larger than 1 . If inequality (4.5b) is violated, then one of the eigenvalues of $B$ is less than -1 . Finally, If inequality $(4.5 \mathrm{c})$ is violated, then $B$ has a complex-conjugate pair of eigenvalues lying outside the unit circle. 
With $B$ defined in model (4.3), it can be shown that inequalities (4.5b) and (4.5c) are always satisfied, and that as $b$ increases, inequality (4.5a) is violated at a critical point $b_{0}$. In terms of the model parameters, and after a bit of rearranging, inequality (4.5a) defined by system (4.3) reads

$$
b<\frac{1-p}{p} \equiv b_{0} .
$$

Thus $b$ must be larger than $b_{0}$ in order for small population to increase from $X=0$.

For the difference equation (4.3) we can also define the intrinsic net reproductive number [10] $R_{0}$ (the average number of offspring that an individuals produces over the course of its lifetime), i.e.,

$$
R_{0}=\frac{b p}{1-p} .
$$

Inequality (4.6) can be rewritten as $R_{0}<1$. That is, if on average, individuals don't replace themselves before they die then the population is doomed.

\subsection{Bifurcations of the positive equilibrium $E^{*}\left(x^{*}, y^{*}\right)$}

The positive equilibrium solution of (4.3) is given as

$$
E^{*}=\left(x^{*}, y^{*}\right)=\left(-\frac{1-p}{1-p q} \ln \left(\frac{1-p}{b p}\right),-\frac{p(1-p)}{1-p q} \ln \left(\frac{1-p}{b p}\right)\right)
$$

with $R_{0}>1$ as a necessary and sufficient condition for a nontrivial equilibrium.

The eigenvalue equation of $B$ at equilibrium $E^{*}$ is

$$
\lambda^{2}-\left[1+p q+(1-p) \ln \left(\frac{1}{R_{0}}\right)\right] \lambda+p q\left[1+(1-p) \ln \left(\frac{1}{R_{0}}\right)\right] .
$$

It is easy to see that $\lambda_{2}=p q$ and $\lambda_{1}=1+(1-p) \ln \left(\frac{1}{R_{0}}\right)$ and $\lambda_{2}$ is less than one in magnitude. If $\lambda_{1}=-1$ we obtain $2+(1-p) \ln \left(\frac{1}{R_{0}}\right)=0$. From this we can conclude that when condition (4.5b) fails, the fixed point becomes unstable through a flip bifurcation at $b=b_{c}=\frac{1-p}{p} \mathrm{e}^{\frac{2}{1-p}}$. Then the main results at the bifurcation may be summarized in the following theorem.

Theorem 4.1. For each fixed $p, q, 0<p<1,0<q<1, E_{0}(0,0)$ will undergo a transcritical bifurcation when

$$
b=b_{0}=\frac{1-p}{p}\left(\text { or } R_{0}=1\right)
$$

and $E^{*}=\left(x^{*}, y^{*}\right)$ will undergo a supercritical fip bifurcation when

$$
b=b_{c}=\frac{1-p}{p} \mathrm{e}^{\frac{2}{1-p}}
$$

Proof. According to the discussion of above, we only need to prove the flip is supercritical. For this purpose, we notice that the Jacobian $D_{E^{*}}$ may be written as

$$
D_{E^{*}}=\left(\begin{array}{c}
(1-p)\left(1+\ln \left(\frac{1}{R_{0}}\right)\right)+p q(1-p)\left(1+\ln \left(\frac{1}{R_{0}}\right)\right) \\
p(1-q)
\end{array}\right) .
$$


Next, we define the matrix

$$
T=\left(\begin{array}{cc}
\frac{\lambda_{1}-p}{p(1-q)} & -1 \\
1 & 1
\end{array}\right)
$$

which columns are the eigenvectors corresponding to the eigenvalues $\lambda_{1}, \lambda_{2}$ of (4.9), and we have

$$
T^{-1}=\left(\begin{array}{cc}
\frac{p(1-q)}{\lambda_{1}-p q} & \frac{p(1-q)}{\lambda_{1}-p q} \\
-\frac{p(1-q)}{\lambda_{1}-p q} & \frac{\lambda_{1}-p}{\lambda_{1}-p q}
\end{array}\right) .
$$

Then after expanding the first component of the model (4.3) up to third order, applying the change of coordinates $(\bar{x}, \bar{y})=\left(x-x^{*}, y-y^{*}\right)$ (in order to translate the bifurcation to the origin) together with the transformations

$$
\left(\begin{array}{l}
\bar{x} \\
\bar{y}
\end{array}\right)=T\left(\begin{array}{l}
u \\
v
\end{array}\right), \quad\left(\begin{array}{l}
u \\
v
\end{array}\right)=T^{-1}\left(\begin{array}{l}
\bar{x} \\
\bar{y}
\end{array}\right),
$$

we can obtain

$$
\left\{\begin{array}{l}
u_{k+1}=\lambda_{1} u_{k}+\frac{1}{a_{11}}\left[-\frac{1}{2}\left(\lambda_{1}+1\right)+p\right] u_{k}^{2}+\frac{1}{6 a_{11}^{2}}\left[\lambda_{1}+2-3 p\right] u_{k}^{3}+o(4), \\
v_{k+1}=\lambda_{2} v_{k}-\frac{1}{a_{11}}\left[-\frac{1}{2}\left(\lambda_{1}+1\right)+p\right] u_{k}^{2}-\frac{1}{6 a_{11}^{2}}\left[\lambda_{1}+2-3 p\right] u_{k}^{3}+o(4),
\end{array}\right.
$$

where $a_{11}=\frac{p(1-q)}{\lambda_{1}-p q}$. In the following we consider the map

$$
(u, v) \rightarrow\left(\lambda_{1} u+g(u, v), \lambda_{2} v+h(u, v)\right),
$$

here $\lambda_{1}=1+(1-p) \ln \left(\frac{1}{R_{0}}\right)(=-1$ at the bifurcation $), \lambda_{2}=p q$, and

$$
\begin{gathered}
g(u, v)=\frac{1}{a_{11}}\left[-\frac{1}{2}\left(\lambda_{1}+1\right)+p\right] u^{2}+\frac{1}{6 a_{11}^{2}}\left[\lambda_{1}+2-3 p\right] u^{3} \\
h(u, v)=-g(u, v) .
\end{gathered}
$$

The next step involves the restriction of (4.11) to the center manifold. Since $g(u, v)$ is independent on $v$. Then, following Guchenheimer and Holmes [12], the center manifold (up to third order) should be

$$
u \rightarrow F(u)=\lambda_{1} u+\frac{1}{a_{11}}\left[-\frac{1}{2}\left(\lambda_{1}+1\right)+p\right] u^{2}+\frac{1}{6 a_{11}^{2}}\left[\lambda_{1}+2-3 p\right] u^{3} .
$$

According to Theorem 3.5.1 (Guchenheimer and Holmes [12]), the bifurcation will be supercritical if the relations

$$
\begin{gathered}
\frac{\partial F}{\partial b} \frac{\partial^{2} F}{\partial u^{2}}+2 \frac{\partial^{2} F}{\partial u \partial b}=\frac{\partial F}{\partial b} \frac{\partial^{2} F}{\partial u^{2}}-\left(\frac{\partial F}{\partial u}-1\right) \frac{\partial^{2} F}{\partial u \partial b} \neq 0, \\
\frac{1}{2}\left(\frac{\partial^{2} F}{\partial u^{2}}\right)^{2}+\frac{1}{3}\left(\frac{\partial^{3} F}{\partial u^{3}}\right)>0
\end{gathered}
$$

hold at the bifurcation. 
(a)

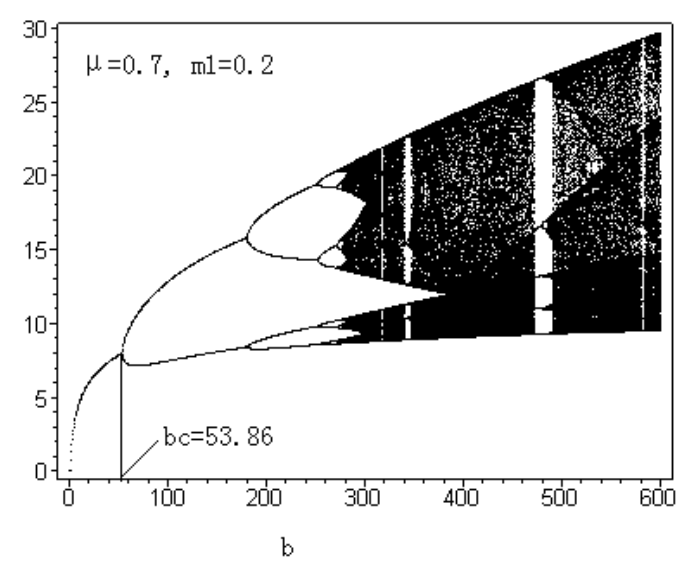

(b)

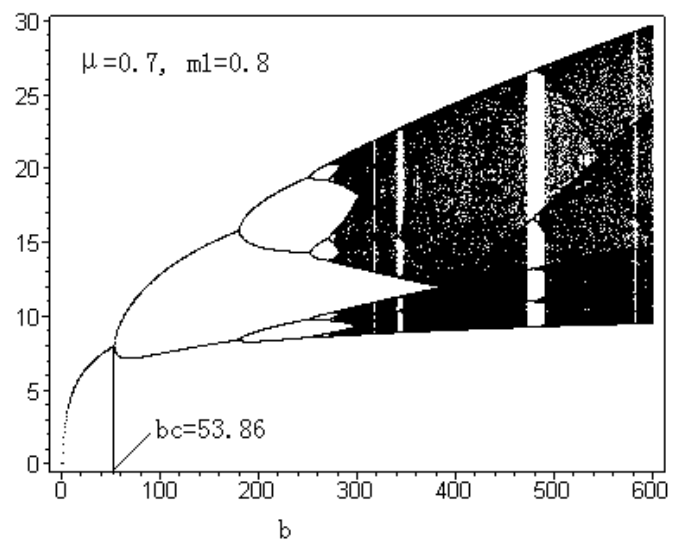

FiguRE 1. Bifurcation diagrams of equation (4.3) for total population. Showing the effect of parameter $q$ on the dynamical behavior of system (4.3). Other parameters are $\mu=0.7, m_{1}=0.2$ in (a) and $\mu=0.7, m_{1}=0.8$ in (b).

To this end it is easy to show that the left-hand side of the nondegeneracy condition (4.12) equals

$$
-\frac{p}{1-p} \mathrm{e}^{-\frac{2}{1-p}}<0
$$

and that (4.13) equals

$$
\frac{(1+p q)^{2}}{p^{2}(1-q)^{2}}\left(2 p^{2}-p+\frac{1}{3}\right)>0
$$

which obviously is valid for all parameters because we have $2 p^{2}-p+\frac{1}{3}=2\left(p-\frac{1}{4}\right)^{2}+\frac{5}{24}>0$. Consequently, the flip is supercritical.

Let us first comment on the flip case. Just beyond the stability threshold $\left(b>b_{c}\right)$ there are stable orbits of period 2, and as shown by numerical experiments. Further increase of $b$ leads through new bifurcations to orbits of period 4,8 , and so on until an accumulation value $b_{a}$ is reached. It should be emphasized that $b_{a}$ is a rather large number compared to $b_{c}$ indeed. If we fix $\mu=0.7, m_{1}=0.2$ (that is $\left.p=\mathrm{e}^{-0.7}, q=\mathrm{e}^{-0.2}\right),\left(x^{*}, y^{*}\right.$ ) undergoes the first flip when $b=b_{c}=53.867$, the 4-periodic orbit emerges as $b$ reaches the value 179.85 and the point of accumulation is found to be as high as $b_{a} \approx 278.11$. This is demonstrated in the bifurcation diagram, Figure 1a.

Remark. From the expressions of $b_{0}, b_{c}$ and Figures. 1a,b we note that the parameter $q(0<q<1)$ does not affect the dynamical behavior of system (4.3).

The bifurcation diagrams (Fig. 2) of model (4.3) reveals another interesting phenomenon. As pointed out above, all of the diagrams are characterized by an alteration of apparently chaotic dynamics and low-period cycles as $b$ increases. Notice that if the cycles to the left of a given chaotic window are of period $k$, then the cycles to the right are of period $k+1$. These so-called "period-adding" sequences have been observed in chemical reactions [11,18] and electrical circuits [20], and have been studied in one-dimensional difference equations [22, 23]. Period-adding is also present in a delay-difference equation population model with density-dependent reproduction [8], and in the density-dependent age-structured model studied by Guckenheimer et al. [13]. 


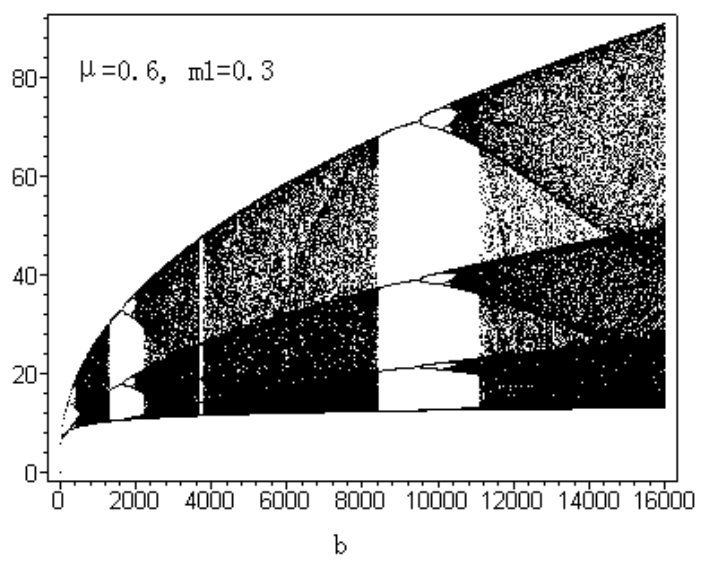

Figure 2. Bifurcation diagrams of equation (4.3) for total population. Showing the periodadding phenomenon. Other parameters are $\mu=0.6, m_{1}=0.3$.

\subsection{The relationships between system (4.1) and equation (4.3)}

In Sections 4.1 and 4.2, we presented the dynamics of system (4.1) using the stroboscopic map. This is a special case of the Poincaré map for periodically forced system or periodically pulsed system; the system trajectory is not recorded continuously in time but once every period of the forcing term or pulsing term (for example, the annual birth pulse period). Long-term solutions of system (4.1) will then appear as follows.

(i) Fixed points of the stroboscopic map (corresponding to periodic solutions having the same period as the pulsing term).

(ii) Periodic points of the stroboscopic map, of period $k$ (corresponding to entrained periodic solutions having exactly $k$ times the period of the pulsing, often called subharmonic periodic solutions or subharmonic period $\left.k^{\prime} s\right)$.

(iii) Invariant circles (corresponding to quasi-periodic solutions, tori $T^{2}$ for the original system of impulsive differential equations).

(iv) Possibly chaotic(strange) attractors.

In the following, we show that the solutions of system (4.1) behave like the above three cases $((i),(i i),(i v))$.

For $b<b_{0}$ (i.e., $\left.R_{0}<1\right)$, equilibrium $E_{0}(0,0)$ is stable. For this range of $b$, trajectories of model (4.1) approach the origin, that is, species goes extinction.

For $b_{0}<b<b_{c}$, the equilibrium $E^{*}$ is stable. For this range of $b$, trajectories of model (4.1) approach the periodic solution $\left(A_{1 p}(t), A_{2 p}(t)\right)$ with period 1 ,

$$
\left\{\begin{array}{l}
A_{1 p}(t)=-\frac{1-p}{p(1-p q)} \ln \left(\frac{1-p}{b p}\right) \mathrm{e}^{-\left(m_{1}+\mu\right)(t-k)}, \\
A_{2 p}(t)=-\frac{1-p}{1-p q} \ln \left(\frac{1-p}{b p}\right) \mathrm{e}^{-\mu(t-k)}\left[1-\mathrm{e}^{-m_{1}(t-k)}\right]-\frac{1-p}{p(1-p q)} \ln \left(\frac{1-p}{b p}\right) \mathrm{e}^{-\mu(t-k)},
\end{array}\right.
$$

where $k \leq t<k+1, p=\mathrm{e}^{-\mu}, q=\mathrm{e}^{-m_{1}}$. That is, periodic solution (4.14) of system (4.1) is locally asymptotically stable. Right at $b=b_{0}$, there is a transcritical bifurcation of periodic solutions as $(0,0)$ and $\left(A_{1 p}(t), A_{2 p}(t)\right)$ pass through each other and exchange stability. Right at $b=b_{c}$, there is a supercritical bifurcation of period-one solution and period-two solution pass through each other and exchange stability. We note in passing that $A_{1 p}(t)$ is discontinuous for $t$ a multiple of $k$ (see Fig. 3).

Corresponding to the bifurcation diagrams in Figure 1a, Figures 3 and 4 illustrate the relationships between model (4.1) and model (4.3) and shows that birth pulse provides a natural period or cyclicity that allows for a period-doubling route to chaos. Figure 3 illustrates a simple cycle of period 1 . Increasing $b$ leads to a cascade of 
(a)

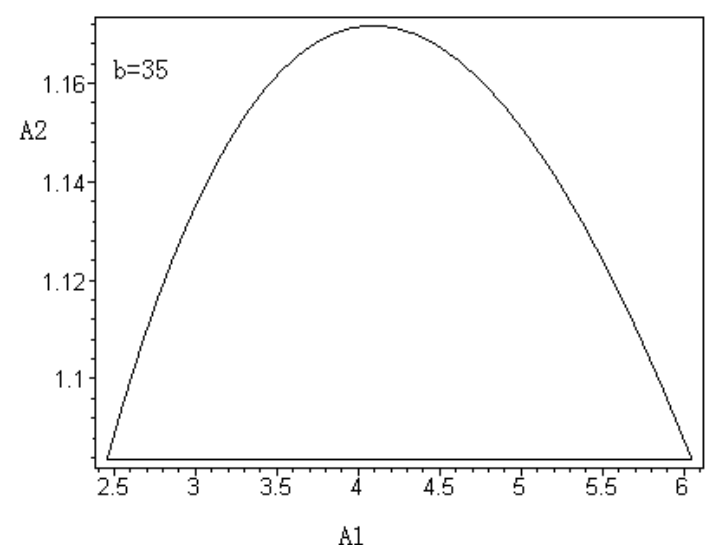

(b)

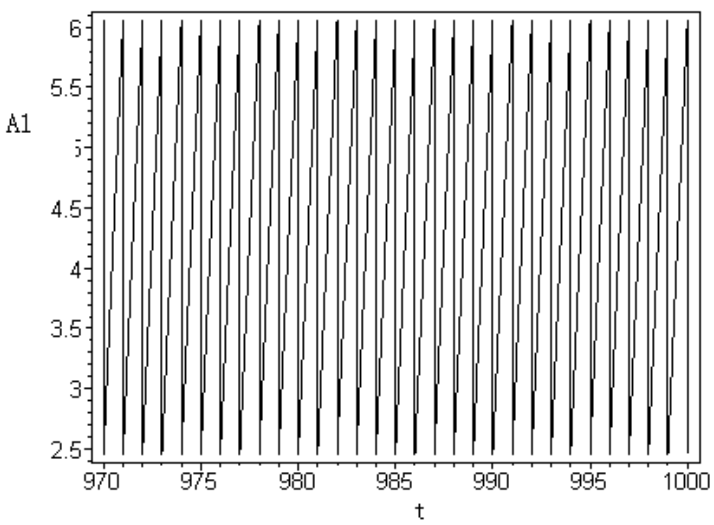

Figure 3. Periodic coexistence of the immature and the mature population with $b=35$, $\mu=0.7, m_{1}=0.2$. (a) period 1 solution; (b) time series for period 1 solution of the immature population.

period-doubling bifurcations (Figs. 4a,b, (a) a 2-period cycle; (b) a 4-period cycle) and finally to the appearance of chaotic strange attractors. Figure $4 \mathrm{c}$ captures one such strange attractor, that is, increasing $b$ is destabilizing: annual oscillations yield to multi-annual cycles of increasing period and amplitude as this parameter is increased (Figs. 3 and 4 ).

\subsection{The one-generation delay case $\boldsymbol{b}_{\mathbf{1}}=\mathbf{0}, \boldsymbol{b}_{\mathbf{2}}=\boldsymbol{b} \neq \mathbf{0}$}

In this section we assume that only mature population can reproduce and study the two-dimensional hybrid matrix model

$$
\left\{\begin{array}{l}
\frac{\mathrm{d} A_{1}(t)}{\mathrm{d} t}=-\left(m_{1}+\mu_{1}\right) A_{1}(t), \\
\frac{\mathrm{d} A_{2}(t)}{\mathrm{d} t}=m_{1} A_{1}(t)-\mu_{2} A_{2}(t), \quad t \neq k \in N, \\
A_{1}\left(k^{+}\right)=A_{1}(k)+b A_{2}(k) \mathrm{e}^{-\left(A_{1}(k)+A_{2}(k)\right)}, \\
A_{2}\left(k^{+}\right)=A_{2}(k), \quad t=k \in N .
\end{array}\right.
$$

For the simplicity, we assume $\mu_{1}=\mu_{2}=\mu$. Similar to system (4.1), we have the following stroboscopic map for system (4.15).

$$
\left\{\begin{array}{l}
A_{1}(k+1)=b p\left[A_{2}(k)+(1-q) A_{1}(k)\right] \mathrm{e}^{-p\left(A_{1}(k)+A_{2}(k)\right)}+p q A_{1}(k), \\
A_{2}(k+1)=p(1-q) A_{1}(k)+p A_{2}(k),
\end{array}\right.
$$

where $p=\mathrm{e}^{-\mu}, q=\mathrm{e}^{-m_{1}}$.

Let $x=p A_{1}, y=p A_{2}$, then system (4.16) becomes as follows

$$
\left\{\begin{array}{l}
x_{k+1}=b p\left[y_{k}+(1-q) x_{k}\right] \mathrm{e}^{-\left(x_{k}+y_{k}\right)}+p q x_{k} \\
y_{k+1}=p(1-q) x_{k}+p y_{k} .
\end{array}\right.
$$

System (4.17) have two equilibria $\bar{E}_{0}(0,0), \bar{E}^{*}\left(\bar{x}^{*}, \bar{y}^{*}\right)$ with

$$
\bar{x}^{*}=-\frac{1-p}{1-p q} \ln \left(\frac{(1-p q)(1-p)}{b p(1-q)}\right), \quad \bar{y}^{*}=-\frac{p(1-p)}{1-p q} \ln \left(\frac{(1-p q)(1-p)}{b p(1-q)}\right) .
$$



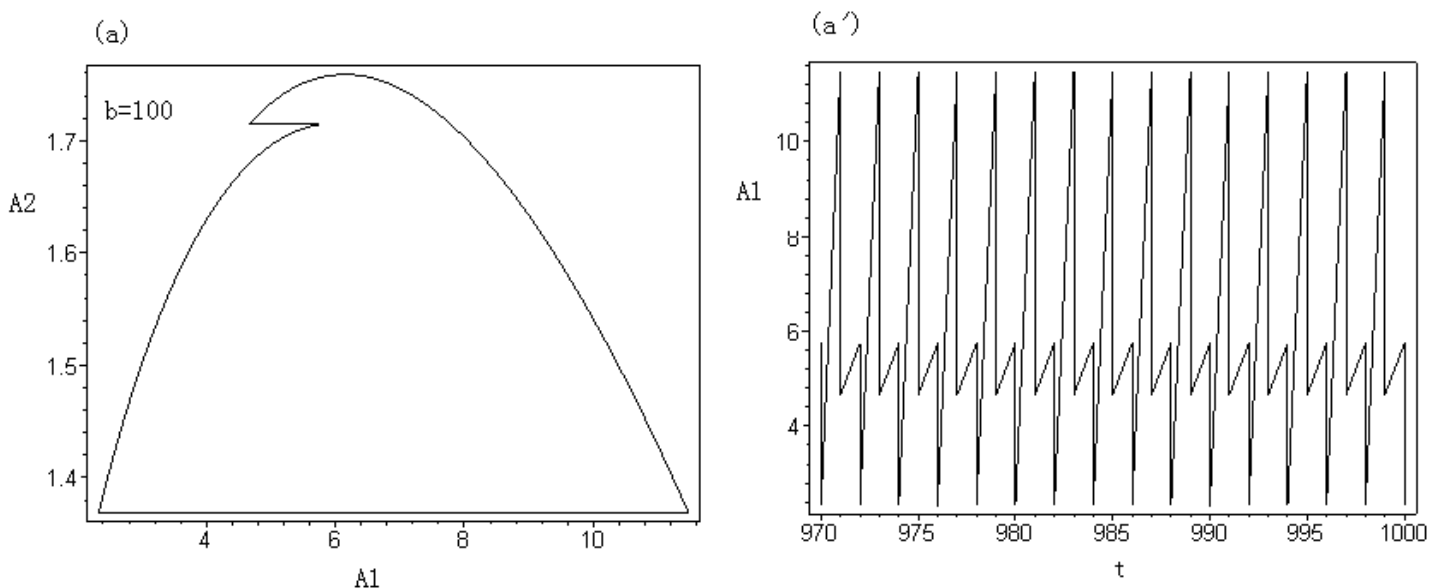

(b)

(b)
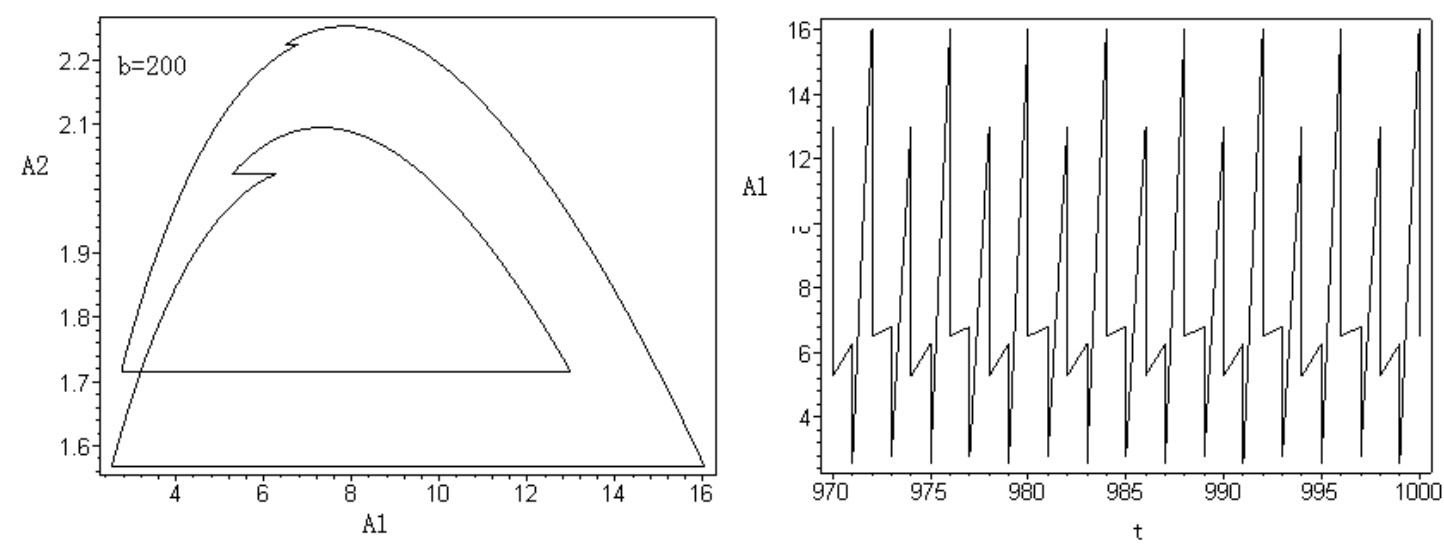

(c)
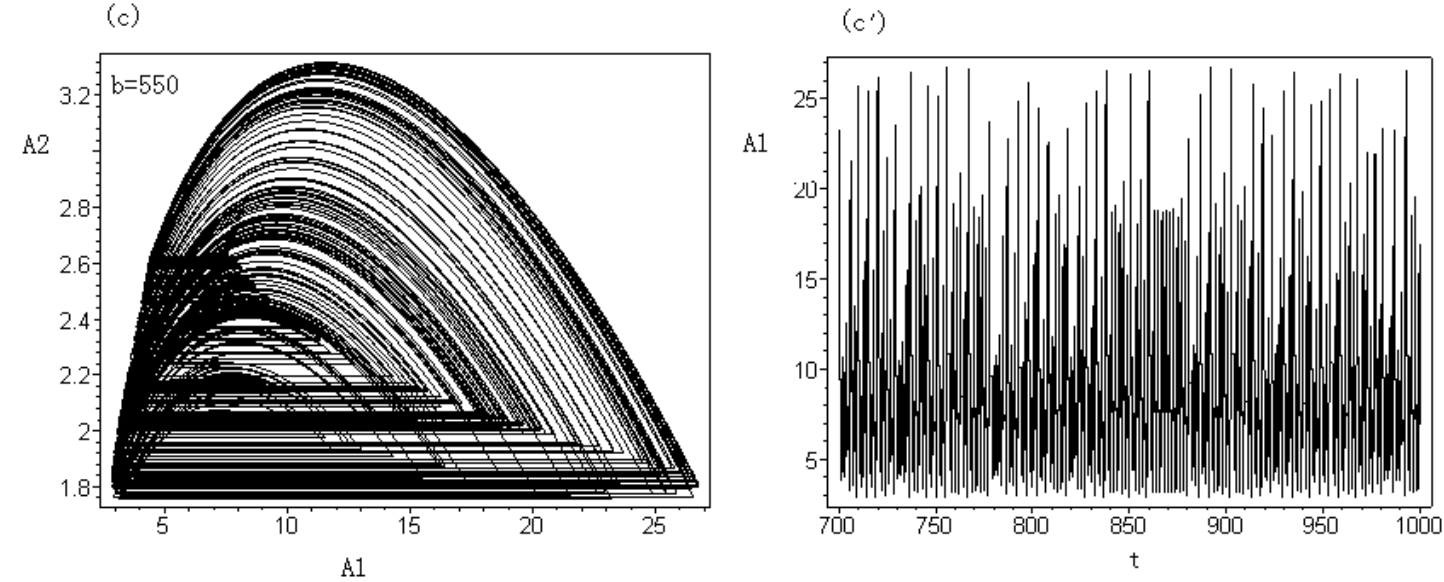

Figure 4. Period-doubling cascade to chaos. (a) a 2-periodic solution, $(a)^{\prime}$, Time series for a 2-periodic solution of immature population; (b) a 4-periodic solution, $(b)^{\prime}$, Time series for a 4-periodic solution of immature population; (c) a strange attractor, $(c)^{\prime}$, Time series for a strange attractor of immature population. Other parameters are $\mu=0.7, m_{1}=0.2$. 
Define $\bar{R}_{0}=\frac{b p(1-p)}{(1-p)(1-p q)}, \bar{b}_{0}=\frac{(1-p)(1-p q)}{p(1-q)}$ and $\bar{b}_{c}=\frac{(1-p)(1-p q)}{p(1-q)} \mathrm{e}^{\frac{2+2 p^{2} q}{1-p)(1+p q)}}$. For system (4.17) we have the same results as those in system (4.3), that is, we have the following theorem

Theorem 4.2. For each fixed $p, q, 0<p<1,0<q<1, \bar{E}_{0}=(0,0)$ will undergo a transcritical bifurcation when

$$
b=\bar{b}_{0}=\frac{(1-p)(1-p q)}{p(1-q)}\left(\text { or } \bar{R}_{0}=1\right),
$$

and $\bar{E}^{*}=\left(\bar{x}^{*}, \bar{y}^{*}\right)$ will undergo a supercritical flip bifurcation when

$$
b=\bar{b}_{c}=\frac{(1-p)(1-p q)}{p(1-q)} \mathrm{e}^{\frac{2+2 p^{2} q}{(1-p)(1+p q)}} .
$$

Proof. We omit the proof of Theorem 4.2 which is similar to the proof of Theorem 4.1.

In the rest of this section, we discuss how the generation delay affects the stability of systems (4.3) and (4.17). For system (4.3), we note that the parameter $q$ does not affect the stability of positive equilibrium $E^{*}$ of system (4.3). Further, from Figures $1 \mathrm{a}$ and $1 \mathrm{~b}$ we know that parameter $q$ does not affect the complexes of system (4.3) either. For this purpose, we shall discuss how parameters $(p, q)$ affect the stability of system in terms of the length $\left(b_{c}-b_{0}\right.$ and $\left.\bar{b}_{c}-\bar{b}_{0}\right)$ of the intervals in which the unique positive equilibria of system (4.3) and (4.17) are stable.

Denote

$$
\begin{gathered}
D(p)=b_{c}-b_{0}=b_{0}\left[\mathrm{e}^{2 /(1-p)}-1\right] \\
\bar{D}(p, q)=\bar{b}_{c}-\bar{b}_{0}=\bar{b}_{0}\left[\mathrm{e}^{\left.\frac{2\left(1+p^{2} q\right)}{(1-p)(1+p q)}-1\right] .}\right.
\end{gathered}
$$

Since

then we have

$$
\begin{aligned}
& \lim _{q \rightarrow 0} \bar{b}_{0}=\lim _{q \rightarrow 0} \frac{(1-p)(1-p q)}{p(1-q)}=\frac{1-p}{p}=b_{0}, \\
& \lim _{q \rightarrow 0} \bar{b}_{c}=\lim _{q \rightarrow 0} \frac{(1-p)(1-p q)}{p(1-q)} \mathrm{e}^{\frac{2\left(1+p^{2} q\right)}{(1-p)(1+p q)}}=b_{c},
\end{aligned}
$$

$$
\lim _{q \rightarrow 0} \bar{D}(p, q)=b_{c}-b_{0}=D(p),
$$

which implies that the stability of the positive equilibria $E^{*}$ and $\bar{E}^{*}$ is uniform as $q \rightarrow 0$.

On the other hand, we have

$$
\frac{\partial \bar{D}(p, q)}{\partial q}=\frac{1-p}{1-q}\left[\frac{1-p}{p(1-q)}\left(\mathrm{e}^{\frac{2\left(1+p^{2} q\right)}{(1-p)(1+p q)}}-1\right)-\frac{2(1-p q)}{(1+p q)^{2}} \mathrm{e}^{\frac{2\left(1+p^{2} q\right)}{(1-p)(1+p q)}}\right] .
$$

Let

$$
f(q)=\frac{1-p}{p(1-q)}\left(\mathrm{e}^{\frac{2\left(1+p^{2} q\right)}{(1-p)(1+p q)}}-1\right)-\frac{2(1-p q)}{(1+p q)^{2}} \mathrm{e}^{\frac{2\left(1+p^{2} q\right)}{(1-p)(1+p q)}} .
$$

Then

$$
\lim _{q \rightarrow 0} f(q)=\frac{1-3 p}{p} \mathrm{e}^{\frac{2}{1-p}}-\frac{1-p}{p} \doteq g(p),
$$

and

$$
\lim _{q \rightarrow 1} f(q)=+\infty .
$$

It is easy to see that the function $g(p)$ satisfies the following property:

(A) There exists $p^{*} \in\left(0, \frac{1}{3}\right)$ such that $g(p)>0$ for $p \in\left(0, p^{*}\right)$, and $g(p)<0$ for $p \in\left(p^{*}, 1\right)$ (see Fig. 5a). 

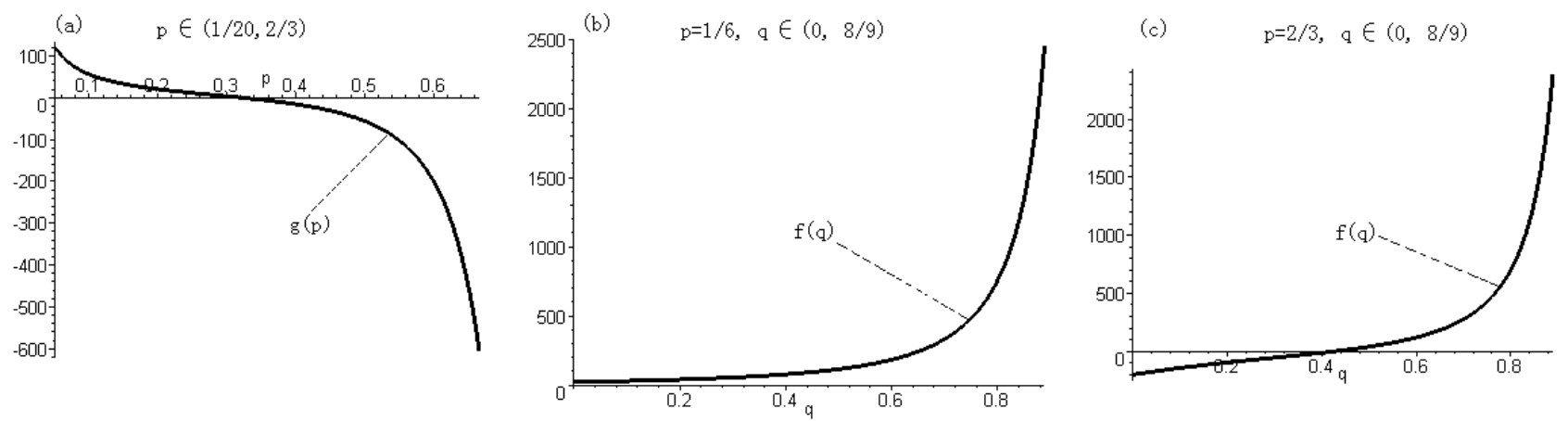

FiguRE 5. The graphes of functions $g(p)$ and $f(q)$. (a) the graph of $g(p)$ for $p \in(1 / 20,2 / 3)$; (b) the graph of $f(q)$ for $p=1 / 6, q \in(0,8 / 9)$; (c) the graph of $f(q)$ for $p=2 / 3, q \in(0,8 / 9)$.
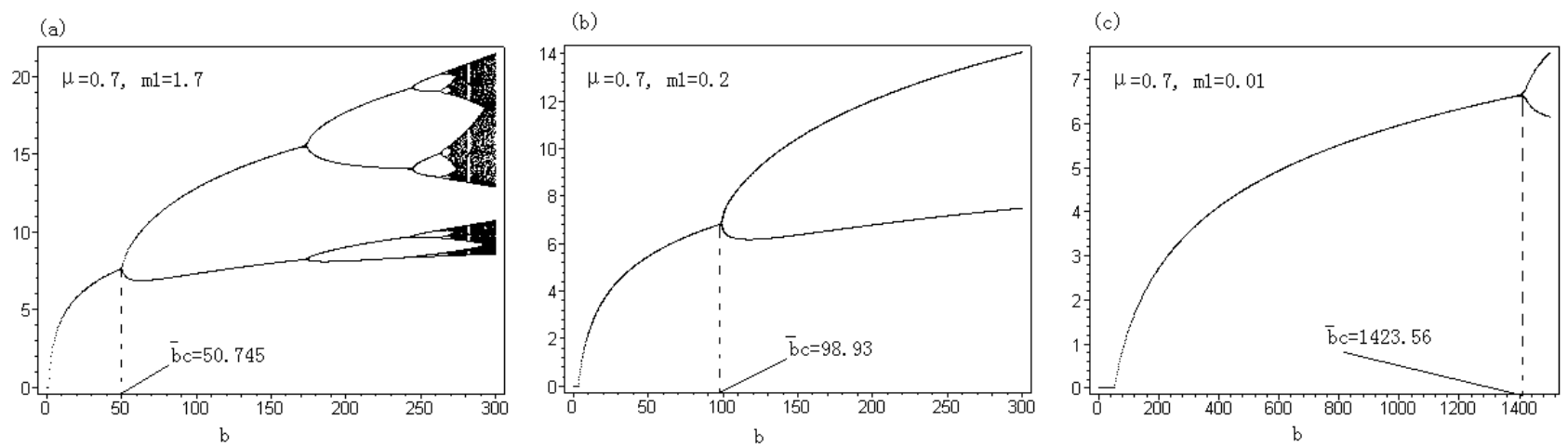

FIgURE 6 . The effect of parameter $q$ or $m_{1}$ on the stability of positive equilibrium. (a) $\mu=0.7$, $m_{1}=1.7, \bar{D}(p, q)=49.61$; (b) $\mu=0.7, m_{1}=0.2, \bar{D}(p, q)=95.60$; (c) $\mu=0.7, m_{1}=0.01$, $\bar{D}(p, q)=1371.77$.

From Figures $5 \mathrm{~b}$ and $5 \mathrm{c}$ we know that $f(q)$ is strictly increased function for any $p \in(0,1)$. By properties $(A)$ and (4.23), we have the following

(1) If $p \in\left(0, p^{*}\right)$, then $f(q)>0$ for all $q \in(0,1)$ (see Fig. $5 \mathrm{~b}$ );

(2) If $p \in\left(p^{*}, 1\right)$, there exists $q^{*} \in(0,1)$ such that $f(q)<0$ for $q \in\left(0, q^{*}\right)$, and $f(q)>0$ for $q \in\left(q^{*}, 1\right)($ see Fig. 5c).

Together with properties $(A),(1)$ and (2) we have the following results on stability of system (4.3) and (4.17).

Result 1. When $p \in\left(0, p^{*}\right)$, we have $\bar{D}(p, q)>D(p)$ for all $q \in(0,1)$ (in this case, we say the stability of equilibrium $E^{*}$ of system (4.3) is weaker than that of system (4.17), i.e., the generation delay strengthens the stability of system).

Result 2. When $p \in\left(p^{*}, 1\right)$, there exists $q^{*} \in(0,1)$ such that $\bar{D}(p, q)<D(p)$ for all $q \in\left(0, q^{*}\right)$ (in this case, we say the stability of equilibrium $E^{*}$ of system (4.3) is stronger than that of system (4.17), i.e., the generation delay weakens the stability of system), and $\bar{D}(p, q)>D(p)$ for all $q \in\left(q^{*}, 1\right)$ (in this case, we say the stability of equilibrium $E^{*}$ of system (4.3) is weaker than that of system (4.17), i.e., the generation delay also strengthens the stability of system).

To illuminate the above results, we give some numerical simulations. In Figure 6 , fix $\mu=0.7$, i.e., $p \approx$ $0.4965 \in\left(p^{*}, 1\right)$. Then $D(0.4965)=52.85$. Let $m_{1}=1.7,0.2,0.01$ (i.e., $\left.q \approx 0.1827,0.8187,0.99\right)$ respectively. 
We have $\bar{D}(0.4965,0.1827)=49.61<D(0.4965)$ (see Fig. 6a), $\bar{D}(0.4965,0.8187)=95.60>D(0.4965)$ (see Fig. $6 \mathrm{~b})$ and $\bar{D}(0.4965,0.99)=1371.77 \gg D(0.4965)$ (see Fig. $6 \mathrm{c}$ ).

\section{Discussion}

We have analyzed what can be considered to be the simplest realistic single species continuous ecosystem models with stage structure subject to periodic birth pulses. Firstly, we have derived our hybrid matrix models from the classical continuous Leslie models, and shown that the trajectories of system are positive invariant. For calculating convenience of the stroboscopic map we have given the analytical solution of system (2.4) between the pulses. In order to understand the dynamical behavior of hybrid matrix models with density-dependent fertility, we restrict our attention to the two-dimensional hybrid matrix models. By using the stroboscopic map and the relationship between the stroboscopic map and hybrid matrix models, we have obtained the complete expression for the periodic solution with period-one and obtained the threshold conditions for its stability. We have presented formal proofs of the supercritical flip bifurcation at the critical value. As the parameter $b$ increases, the solution may pass from a stabilize period-one (annual) cycle to a stabilize period-two cycle to a period-four (multi-annual cycle), etc., tending to a Feigenbaum transition to chaotic behavior, which show that the dynamical behavior of hybrid matrix models are very complex as parameters varies.

Comparing systems (4.1) and (4.15) with corresponding to continuous age-structured models (that is, systems (4.1) and (4.15) do not have impulsive effects), we can conclude that continuous age-structured models are, as we have known, dominated by its equilibria $[10,28,31]$. It possesses two equilibria, corresponding to washout of population and coexistence. These equilibria are the only feasible attractors. Systems (4.1) and (4.15), in contrast, are dominated by periodic and by chaotic dynamics. So we can conclude that the periodic birth pulsing of the population (a) destroys equilibria, (b) introduces subharmonic synchronization, and (c) initiates chaos. So we can conclude that pulsing, in effect, provides a natural period of cyclicity that allows for a period-doubling route to chaos.

One of the most significant results obtained from the previous section was that the generation delay may act as both a stabilizing and a destabilizing effect. In fact, our results are as follows. If the death rate of population satisfies $\mu \in\left(-\ln \left(p^{*}\right), 1\right)$ (where $p^{*} \in(0,1 / 3)$ ), then the generation delay strengthens the stability of period-1 solution, at the same time the maturation rate does not affect the stability of period- 1 solution when the death rate is relatively high. If $\mu \in\left(0,-\ln \left(p^{*}\right)\right)$, we find a critical value of maturation rate $m_{1}^{*}=-\ln \left(q^{*}\right)\left(q^{*} \in\right.$ $(0,1))$ such that the ganeration delay weakens the stability of period-1 solution as $m_{1} \in\left(-\ln \left(q^{*}\right), 1\right)$, while it strengthens the stability of periodic solution as $m_{1} \in\left(0,-\ln \left(q^{*}\right)\right)$. This shows the effect of generation delay on the stability of period-1 solution is dependent on the maturation rate when the death rate is relatively small.

Another aspect which also should be mentioned is when the density-dependent fertility is considered into the models, the discrete dynamical system determined by the stroboscopic map becomes nonlinear, then the population in the pulsed birth time is characterized by the existence and stability of equilibria, by the bifurcations that occur when stability is lost, and by the patterns of dynamics (cycles, chaos) that follow the bifurcations, which imply that the density-dependent fertility make the hybrid matrix models more complex than those of the continuous age-structured models $[6,7,9,10]$.

Many authors have made experiments in order to investigate how stage structure influences the population fluctuations. Laboratory insect cultures in which the critical controlling factor is the supply of larval food $[32,33]$ often display large quasi-cyclic population fluctuations which the period of the cycles is 2-3 times the maturation time. The experiments by Lawton on populations of Indian meal moth Plodia interpunctella [14] have reemphasized that cycles with periods close to the generation time are also possible. Our theoretical results in this paper show that periodic birth pulses make single species model with stage structure occur with varies kinds of periodic fluctuations, such as annual cycles and multi-annual cycles, which are in accord with the above experiments. This suggests that it is more in line with reality from a biological point of view when we consider the mature population with an annual birth pulse.

From [10], we note that it is easy to obtain the conditions under which species for $n$-dimensional discrete Leslie matrix models go to extinction or persistence. However, it is very difficult for us to obtain the conditions 
under which guarantee the extinction or persisitence of species for n-dimensional hybrid matrix models (2.4), even if we assume all parameters are constants, which need further exploration.

\section{REFERENCES}

[1] Z. Agur, L. Cojocaru, R. Anderson and Y. Danon, Pulse mass measles vaccination across age cohorts. Proc. Natl. Acad. Sci. USA 90 (1993) 11698-11702.

[2] W.G. Aiello and H.I. Freedman, A time delay model of single-species growth with stage structure. Math. Biosci. 101 (1990) $139-153$.

[3] W.G. Aiello, H.I. Freedman and J. Wu, Analysis of a model representing stage structured population growth with statedependent time delay. SIAM J. Appl. Math. 52 (1990) 855-869.

[4] D.D. Bainov and P.S. Simeonov, System with impulsive effect: stability, theory and applications. John Wiley \& Sons, New York (1989).

[5] J.R. Bence and R.M. Nisbet, Space limited recruitment in open systems: The importance of time delays. Ecology 70 (1989) $1434-1441$

[6] O. Bernard and J.L. Gouzé, Transient behavior of biological loop models, with application to the droop model. Math. Biosci. 127 (1995) 19-43.

[7] O. Bernard and S. Souissi, Qualitative behavior of stage-structure populations: application to structure validation. J. Math. Biol. 37 (1998) 291-308.

[8] L.W. Botsford, Further analysis of Clark's delayed recruitment model. Bull. Math. Biol. 54 (1992) $275-293$.

[9] J.M. Cushing, Equilibria and oscillations in age-structured population growth models, in Mathematical modelling of environmental and ecological system, J.B. Shukla, T.G. Hallam and V. Capasso Eds., Elsevier, New York (1987) 153-175.

[10] J.M. Cushing, An introduction to structured population dynamics. CBMS-NSF Regional Conf. Ser. in Appl. Math. 71 (1998) $1-10$.

[11] I.R. Epstein, Oscillations and chaos in chemical systems. Phys. D 7 (1983) 47-56.

[12] J. Guckenheimer and P. Holmes, Nonlinear oscillations, dynamical systems and bifurcations of vector fields. Springer Verlag, Berlin, Heidelberg, New York, Tokyo (1990).

[13] J. Guckenheimer, G. Oster and A. Ipaktchi, The dynamics of density dependent population models. J. Math. Biol. 4 (1977) $101-147$.

[14] W.S.C. Gurney, R.M. Nisbet and J.L. Lawton, The systematic formulation of tractable single-species population models incorporating age-structure. J. Anim. Ecol. 52 (1983) 479-495.

[15] W.S.C. Gurney, R.M. Nisbet and S.P. Blythe, The systematic formulation of model of predator prey populations. Springer, J.A.J. Metz and O. Dekmann Eds., Berlin, Heidelberg, New York, Lecture Notes Biomath. 68 (1986).

[16] A. Hastings, Age-dependent predation is not a simple process. I. continuous time models. Theor. Popul. Biol. 23 (1983) $347-362$.

[17] S.P. Hastings, J.J. Tyson and D. Webster, Existence of periodic solutions for negative feedback cellular control systems. J. Differential Equations 25 (1977) 39-64.

[18] M.J.B. Hauser, L.F. Olsen, T.V. Bronnikova and W.M. Schaffer, Routes to chaos in the peroxidase-oxidase reaction: perioddoubling and period-adding. J. Phys. Chem. B 101 (1997) 5075-5083.

[19] S.M. Henson, Leslie matrix models as "stroboscopic snapshots" of McKendrick PDE models. J. Math. Biol. 37 (1998) 309-328.

[20] Y.F. Hung, T.C. Yen and J.L. Chern, Observation of period-adding in an optogalvanic circuit. Phys. Lett. A 199 (1995) 70-74.

[21] E.I. Jury, Inners and stability of dynamic systems. Wiley, New York (1974).

[22] K. Kaneko, On the period-adding phenomena at the frequency locking in a one-dimensional mapping. Progr. Theoret. Phys. 69 (1982) 403-414.

[23] K. Kaneko, Similarity structure and scaling property of the period-adding phenomena. Progr. Theoret. Phys. 69 (1983) 403-414.

[24] M.J. Kishi, S. Kimura, H. Nakata and Y. Yamashita, A biomass-based model for the sand lance in Seto Znland Sea. Japan. Ecol. Model. 54 (1991) 247-263.

[25] A. Lakmeche and O. Arino, Bifurcation of non trivial periodic solutions of impulsive differential equations arising chemotherapeutic treatment. Dynam. Contin. Discrete Impuls. Systems 7 (2000) 165-287.

[26] V. Laksmikantham, D.D. Bainov and P.S. Simeonov, Theory of impulsive differential equations. World Scientific, Singapore (1989).

[27] P.H. Leslie, Some further notes on the use of matrices in certain population mathematics. Biometrika 35 (1948) $213-245$.

[28] S.A. Levin, Age-structure and stability in multiple-age spawning populations. Springer-Verlag, T.L. Vincent and J.M. Skowrinski Eds., Berlin, Heidelberg, New York, Lecture Notes Biomath. 40 (1981) 21-45.

[29] S. A.Levin and C.P. Goodyear, Analysis of an age-structured fishery model. J. Math. Biol. 9 (1980) $245-274$.

[30] T. Lindstrom, Dependencies between competition and predation-and their consequences for initial value sensitivity. SIAM J. Appl. Math. 59 (1999) 1468-1486. 
[31] J.A.J. Metz and O. Diekmann, The dynamics of physiologically structured populations. Springer, Berlin, Heidelberg, New York, Lecture notes Biomath. 68 (1986).

[32] A.J. Nicholson, An outline of the dynamics of animal populations. Aust. J. Zool. 2 (1954) 9-65.

[33] A.J. Nicholson, The self adjustment of populations to change. Cold Spring Harbor Symp. Quant. Biol. 22 (1957) 153-173.

[34] J.C. Panetta, A mathematical model of periodically pulsed chemotherapy: tumor recurrence and metastasis in a competition environment. Bull. Math. Biol. 58 (1996) 425-447.

[35] B. Shulgin, L. Stone and Z. Agur, Pulse vaccination strategy in the SIR epidemic model. Bull. Math. Biol. 60 (1998) 1-26.

[36] S.Y. Tang and L.S. Chen, Density-dependent birth rate, birth pulses and their population dynamic consequences. J. Math. Biol. 44 (2002) 185-199.

[37] G. Uribe, On the relationship between continuous and discrete models for size-structured population dynamics. Ph.D. dissertation, Interdisciplinary program in applied mathematics, University of Arizona, Tucson, USA (1993).

To access this journal online:

www.edpsciences.org 